\title{
Incidence of venous thromboembolism in psychiatric inpatients: a chart review
}

This article was published in the following Dove Press journal:

Neuropsychiatric Disease and Treatment

\author{
Masahiro Takeshima ${ }^{1, *}$ \\ Hiroyasu Ishikawa ${ }^{1,2, *}$ \\ Kazumi Shimizu ${ }^{3}$ \\ Takashi Kanbayashi' \\ Tetsuo Shimizu' \\ 'Department of Neuropsychiatry, \\ Akita University Graduate School of \\ Medicine, Akita, Japan; ${ }^{2}$ Department \\ of Neuropsychiatry, Nakadori \\ Rehabilitation Hospital, Akita, \\ Japan; ${ }^{3}$ Palliative Care Center, Akita \\ University Hospital, Akita, Japan \\ *These authors contributed equally \\ to this work
}

Purpose: Venous thromboembolism (VTE) is the combination of pulmonary embolism (PE) and deep vein thrombosis. In recent years, VTE has been gaining attention in the field of psychiatry as it can cause sudden deaths in patients hospitalized in psychiatric departments. The purpose of this study was to investigate the incidence of VTE in psychiatric inpatients using contrastenhanced computed tomography (CT).

Patients and methods: At the psychiatric department of the Akita University Hospital, NANOPIA ${ }^{\circledR}$ D-dimer was measured in patients with suspected symptomatic VTE or believed to be at risk for asymptomatic VTE. A follow-up contrast-enhanced CT was also performed in cases of D-dimer values over $1 \mu \mathrm{g} / \mathrm{mL}$. Patients diagnosed with VTE based on contrast-enhanced CT during hospitalizations between May 1, 2009 and April 30, 2017 were analyzed. VTE incidence was compared in restrained and unrestrained catatonic and noncatatonic patients. We also investigated whether VTE was symptomatic or asymptomatic as well as its outcomes.

Results: The overall incidence of VTE was $2.3 \%(39 / 1,681)$ in the 8 -year period. VTE was observed in $61.1 \%(11 / 18)$ of catatonic patients, $4.1 \%(11 / 270)$ of noncatatonic restrained patients, and $1.2 \%(17 / 1,393)$ of noncatatonic unrestrained patients. PE was observed in $76.9 \%$ $(30 / 39)$ of VTE patients and $97.4 \%$ (38/39) of VTE patients were asymptomatic. Recovery was achieved in all cases of VTE treated with anticoagulation therapy.

Conclusion: These results indicate that the risk of VTE is high in psychiatric inpatients and that PE is common in these population. The data may also suggest that contrast-enhanced CT is important in surveying thrombus in suspected cases of VTE. In the psychiatric field, proper attention must be given to VTE, regardless of the presence or absence of catatonia or restraint, particularly given that PE was observed in more than $75 \%$ of cases of VTE.

Keywords: computed tomography, deep vein thrombosis, D-dimer, psychiatric patients, catatonia, pulmonary embolism

\section{Introduction}

Venous thromboembolism (VTE), a collective name for the combination of pulmonary embolism (PE) and deep vein thrombosis (DVT), has been gaining attention in recent years as a risk factor for sudden death in patients hospitalized in psychiatric departments. ${ }^{1}$ Various reports have emerged with the following findings: $4 \%$ of unexpected sudden deaths in psychiatric patients resulted from $\mathrm{PE},{ }^{2}$ antipsychotic medications increased the risk of VTE, ${ }^{3,4} 11.6 \%$ of patients who are physically restrained developed DVT, ${ }^{5}$ and $25.3 \%$ of catatonic patients had DVT. ${ }^{6}$

To the present day, systematic research on VTE in psychiatric inpatients has been limited to catatonic and restrained patients. Moreover, past reports have investigated the presence of DVT using lower limb venous ultrasonography, and contrast-enhanced computed tomography (CT) has not been used in the examination of VTE cases that
Correspondence: Masahiro Takeshima Department of Neuropsychiatry, Akita University Graduate School of Medicine, I-I-I, Hondo, Akita-shi, Akita 010-8543, Japan

Tel $+81 \quad 188846122$

Fax +81188846445

Emailm.takeshima@med.akita-u.ac.jp

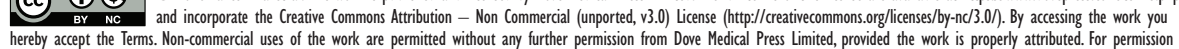
for commercial use of this work, please see paragraphs 4.2 and 5 of our Terms (https://www.dovepress.com/terms.php). 
include PE. ${ }^{2-6}$ Therefore, we conducted a retrospective study to investigate the incidence of VTE (PE as well as DVT) in psychiatric inpatients, regardless of the presence or absence of catatonia or restraint.

\section{Patients and methods}

\section{Study design}

This study was a retrospective epidemiologic study to investigate the incidence of VTE in psychiatric inpatients.

\section{Study setting and population}

This study included consecutive cases hospitalized in the psychiatric department of Akita University Hospital during an 8-year period between May 1, 2009 and April 30, 2017. The psychiatric department of Akita University Hospital is an acute medical unit with 36 beds. In 2016, the average length of stay was 30.7 days in the psychiatry department of Akita University. When a patient was suspected of having symptomatic VTE or believed to be at high risk for asymptomatic VTE by the attending physician because of restraint, catatonia, decreased motivation/activity, or other factors, NANOPIA ${ }^{\circledR}$ D-dimer was measured routinely. In cases of an abnormal D-dimer value ( $1 \mu \mathrm{g} / \mathrm{mL}$ or more), contrast-enhanced CT was performed based on the attending physician's judgment. Furthermore, as a rule at our hospital, contrast-enhanced CT was performed in suspected cases of VTE, without the requirement for lower limb venous ultrasonography.

We targeted 2,468 serial cases and analyzed 1,681 cases as illustrated in Figure 1. Patients who were hospitalized for short-term periods (under 1 week) during the study period were excluded because of insufficient clinical information. In brief, patients hospitalized up to 3 days for. continuouselectroconvulsive therapy (C-ECT) or those hospitalized within a week for nonpsychiatric medical problems were excluded. We then assessed the number of patients subjected to NANOPIA D-dimer test as a screening test for VTE during the study period, the number of patients with abnormal NANOPIA D-dimer levels (above $1 \mu \mathrm{g} / \mathrm{mL}$ ), the number of patients for which contrast-enhanced CT was performed for the diagnosis of VTE, and the number of patients diagnosed with VTE based on the results of contrast-enhanced CT. Of the limited cases in which contrast-enhanced CT data $(n=101)$ were available, patients were classified into 1 of 3 groups: catatonic patients, noncatatonic restrained patients, and noncatatonic unrestrained patients. Cases of NANOPIA D-dimer values below $1 \mu \mathrm{g} / \mathrm{mL}$ were judged as VTE-negative. Catatonic patients were diagnosed according to the Diagnostic and Statistical Manual of Mental Disorders, Fifth Edition criteria based on the description from electrical medical charts. ${ }^{7}$

The following parameters were assessed in cases in which contrast-enhanced CT was performed: age; sex; body mass

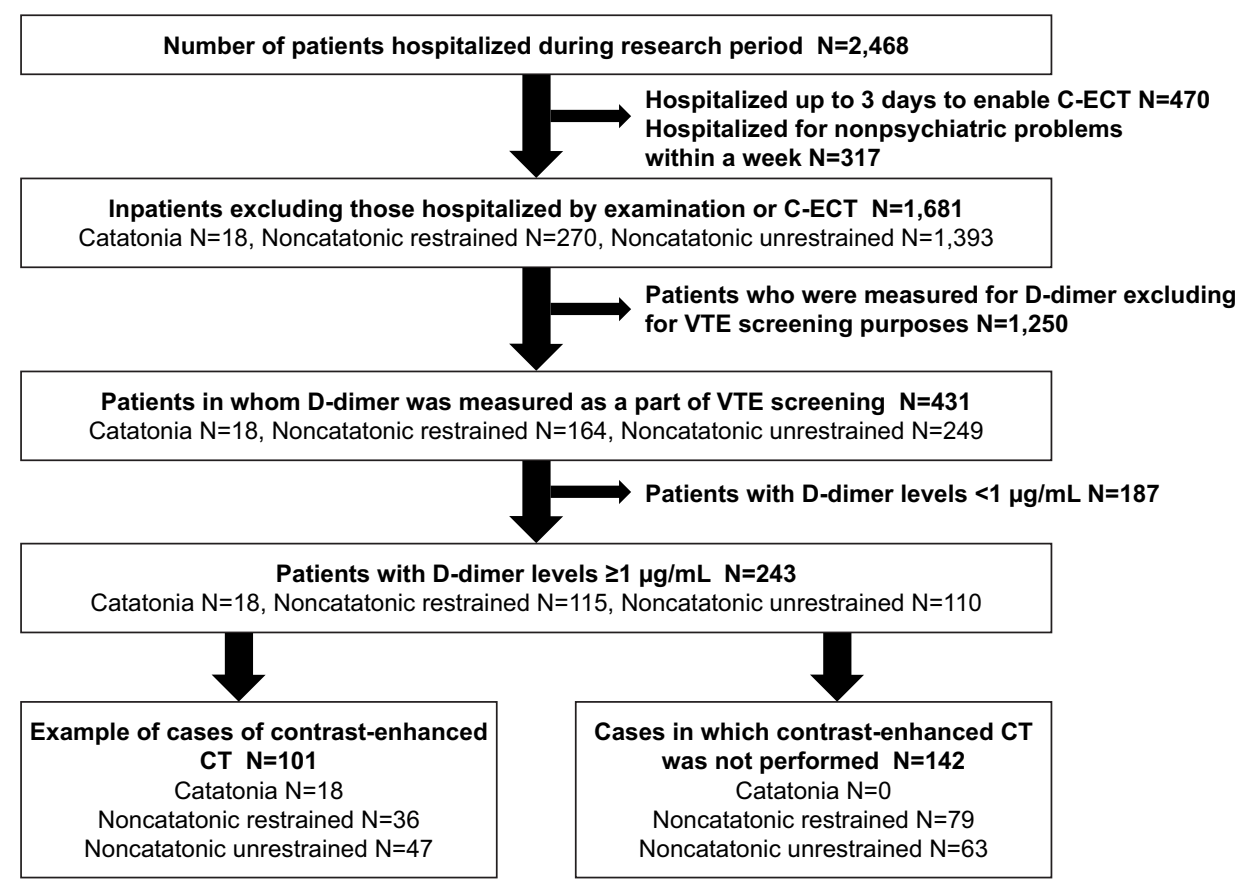

Figure I Selection of participants.

Notes: After applying the inclusion and exclusion criteria, there were I,68I hospitalizations. There were 431 patients who underwent D-dimer assessment as part of the VTE screening, 243 patients with D-dimer levels $\geq 1 \mu \mathrm{g} / \mathrm{mL}$, and $10 \mathrm{I}$ patients who underwent contrast-enhanced CT.

Abbreviations: C-ECT, continuous-electroconvulsive therapy; CT, computed tomography; VTE, venous thromboembolism. 
index $(\mathrm{BMI})\left(\mathrm{kg} / \mathrm{m}^{2}\right)$; D-dimer value $(\mu \mathrm{g} / \mathrm{mL})$; catatonia; duration of restraint (days); duration of restraint of lower extremities (days); prevention of VTE using graduated compression stockings (GCS), intermittent pneumatic compression (IPC), or heparin administration; history of VTE; diagnosis of psychiatric disorder (International Classification of Diseases 10th Revision: ICD-10); daily dosage of antipsychotics; and use of antidepressants. The daily dosage of antipsychotics on the day when D-dimer was measured for the VTE screening was converted to chlorpromazine equivalents (CPZE). The subjects who were administered antipsychotics were divided by CPZE into 4 groups: $0 \mathrm{mg} /$ day, $>0 \mathrm{mg}$ and $<300 /$ day, $\geq 300 \mathrm{mg} /$ day and $<600 \mathrm{mg} /$ day, or $\geq 600 \mathrm{mg} /$ day. The daily dosage of antidepressants on the day when D-dimer was measured for VTE screening was converted to imipramine equivalents (IMPE). The subjects who were administered antidepressants were divided by IMPE into 3 groups: $0 \mathrm{mg} /$ day, $>0 \mathrm{mg}$ and $<150 /$ day, or $\geq 150 \mathrm{mg} /$ day. The following parameters were also assessed: malignancy, surgery within 4 weeks, trauma within 4 weeks, diabetes, pregnancy, oral contraceptive use, and hormone replacement therapy. The amount of time (hours) from D-dimer measurement to contrast-enhanced CT was examined. The closest D-dimer value from the contrast-enhanced CT was adopted. Contrastenhanced CT-diagnosed VTE cases were examined based on classifications of PE + DVT, PE only, and DVT only. Assessment of VTE cases included the outcome and whether the case was symptomatic or asymptomatic. Type of DVT was determined as either proximal or distal type. Proximal/distal DVT was defined as when the thrombus in the iliac, femoral, or popliteal veins was at the distal aspect of the popliteal vein.

\section{D-dimer}

The plasma levels of D-dimer were measured using NANOPIA (Sekisui Medical Company Limited, Tokyo Japan), a commercially available latex agglutination assay kit, and the results were expressed as micrograms per milliliter $(\mu \mathrm{g} / \mathrm{mL})$. The latex agglutination assay uses latex microparticles coated with a monoclonal antibody specific for fragment D-dimer. A positive test result was defined as a D-dimer level $\geq 1.0 \mu \mathrm{g} / \mathrm{mL}^{8}{ }^{8}$ The measuring equipment used an automated coagulation analyzer COAPRESTA 2000 (Sekisui Medical Co., Ltd., Tokyo, Japan). VTE can be excluded when NANOPIA D-dimer values are $<1 \mu \mathrm{g} / \mathrm{mL}{ }^{8}$

\section{Computed tomography}

CT scans for detecting VTE (pulmonary arterial embolization or deep venous thrombosis) were performed using multidetector row helical CT scanners (Discovery CT750HD or
Discovery CT750HD-A; GE Healthcare Japan, Tokyo, Japan) with intravenously injected low-osmolar iodinated contrast medium (Omnipaque 300; Daiichi Sankyo Company Limited, Tokyo, Japan). Slice thickness was $1.25 \mathrm{~mm}$. Total amount of contrast medium was determined as "body weight $(\mathrm{kg}) \times 2+$ 30 (mL)", up to $150 \mathrm{~mL}$. Contrast medium was intravenously injected at a rate of $4.0 \mathrm{~mL} / \mathrm{s}$ using a power injector, and scanning was performed at 20 seconds (early phase) for detecting pulmonary arterial embolization and at 210 seconds (delayed phase) for detecting DVT. CT scans were analyzed by radiological-diagnositc specialists of the Japan Radiological Society or doctors specializing in diagnosis who had worked exclusively in a radiology department for over 10 years.

\section{Data analysis}

The data were expressed as mean $\pm \mathrm{SD}$ for age and BMI. The data were expressed as median $(25 \%-75 \%$ percentile) for D-dimer. To examine the differences between VTE and nonVTE patients, a chi-squared test was performed for age, sex, BMI, catatonia, duration of restraint, diagnosis of psychiatric disorder (ICD-10 score), antipsychotics dosage, antidepressants dosage, and diabetes. Fisher's exact test was performed for duration of restraint of the lower extremities, prevention of VTE, history of VTE, malignancy, surgery within 4 weeks, and trauma within 4 weeks. To examine the differences between VTE and non-VTE, a Mann-Whitney $U$-test was used for the D-dimer value. To examine which factor(s) predict VTE considering possible intercorrelations for sex, age (ie, $<65, \geq 65$ years), catatonia, and duration of restraint (ie, 0 day, $\geq 1$ day, and $\leq 6$ or $\geq 7$ days), a multiple logistic regression analysis was performed, and the odds ratio was estimated. All statistical analyses were performed with EZR (Saitama Medical Center, Jichi Medical University, Saitama, Japan), a graphical user interface for R 2.13.0 (R Foundation for Statistical Computing, Vienna, Austria). ${ }^{9}$ More precisely, EZR is a modified version of $\mathrm{R}$ commander (version 1.6-3) designed to add statistical functions frequently used in biostatistics. ${ }^{9}$

\section{Ethical issues}

This study was approved by the Ethical Committee for Human Research of Akita University (No 1822), who also exempted the study from the requirement of informed consent because the study involved de-identified data acquired during routine care of the patient.

\section{Results}

\section{Demographics}

Selection of participants in this study is shown in Figure 1. Throughout the study period, there were 2,468 hospitalized 
cases, all of whom were Japanese. There were 470 cases of short-term hospitalization up to 3 days for C-ECT and 317 cases of short-term hospitalization ( 7 days) for examination of nonpsychiatric medical problems. With the exclusion of these inpatients, 1,681 cases remained throughout the research period. There were 18 cases of catatonia, 270 cases of noncatatonic restrained, and 1,393 cases of noncatatonic unrestrained of the 1,681 hospitalizations during the 8 -year period. There were 431 cases of D-dimer being measured at the VTE screening, further categorized as 18 cases of catatonia, 164 cases of noncatatonic restrained, and 249 cases of noncatatonic unrestrained. There were 243 cases of abnormal D-dimer values, with a breakdown of 18 cases of catatonia, 115 cases of noncatatonic restrained, and 111 cases of noncatatonic unrestrained. Contrast-enhanced CT was performed on 101 cases of abnormal D-dimer

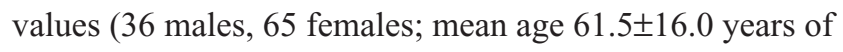
age), with a breakdown of 18 cases of catatonia, 36 cases of noncatatonic restrained, and 47 cases of noncatatonic unrestrained. Ninety cases had contrast-enhanced CT performed within 24 hours of the D-dimer measurement, and 11 cases had contrast-enhanced CT performed within 72 hours of the D-dimer measurement. There were no occurrences of lower limb ultrasonography examinations prior to contrastenhanced CT in cases of abnormal D-dimer values.

VTE was observed in $2.3 \%(39 / 1,681)$ of all patients, in $61.1 \%(11 / 18)$ of catatonic patients, $4.1 \%(11 / 270)$ of noncatatonic restrained patients, and $1.2 \%$ (17/1,393) of noncatatonic unrestrained patients. VTE was identified in $38.6 \%$ $(39 / 101)$ of all patients who underwent contrast-enhanced $\mathrm{CT}$, in $61.1 \%(11 / 18)$ of catatonic patients, in $30.6 \%(11 / 36)$ of noncatatonic restrained patients, and in $36.2 \%(17 / 47)$ of noncatatonic unrestrained patients.

Concerning the diagnosis of VTE, 28.2\% (11/39) of VTE patients demonstrated a co-occurrence of PE and DVT, $48.7 \%$ (19/39) of VTE patients demonstrated the presence of PE only, and $23.1 \%$ (9/39) of VTE patients demonstrated the presence of DVT only. In addition, 48.7\% (19/39) of VTE patients had PE without DVT (Table 1), and 97.4\% (38/39) of VTE patients were asymptomatic for VTE. One symptomatic patient was diagnosed as PE. In 55.0\% (11/20) of patients, the DVT was located proximally. Anticoagulant therapy was performed in all VTE events and recovery was gained in all cases.

Of the 101 patients who underwent contrast-enhanced $\mathrm{CT}$, there were 36 men and 65 women. The mean age was $61.5 \pm 16.0$ years, and mean BMI was $21.4 \pm 5.3 \mathrm{~kg} / \mathrm{m}^{2}$. The median D-dimer value was $7.56 \mu \mathrm{g} / \mathrm{mL}$ (25th percentile
Table I Details of VTE-positive cases

\begin{tabular}{lllll}
\hline $\begin{array}{l}\text { Patients } \\
\text { with }\end{array}$ & $\begin{array}{l}\text { Catatonia } \\
\text { patients, } \\
\text { VTE }\end{array}$ & $\begin{array}{l}\text { Noncatatonic } \\
\text { restrained } \\
\text { patients, } \\
\text { N=I I (\%) }\end{array}$ & $\begin{array}{l}\text { Noncatatonic } \\
\text { unrestrained } \\
\text { patients, } \\
\mathbf{N}=\text { I I (\%) }\end{array}$ & $\begin{array}{l}\text { Total, } \\
\mathbf{N}(\%)\end{array}$ \\
\hline PE + DVT & $3(7.7)$ & I (2.6) & $7(17.9)$ & II (28.2) \\
PE only & $5(12.8)$ & $8(20.5)$ & $6(15.4)$ & $19(48.7)$ \\
DVT only & $3(7.7)$ & $2(5.1)$ & $4(10.3)$ & $9(23.1)$ \\
\hline
\end{tabular}

Abbreviations: $\mathrm{DVT}$, deep vein thrombosis; $\mathrm{PE}$, pulmonary embolism; $\mathrm{VTE}$, venous thromboembolism.

$3.61 \mu \mathrm{g} / \mathrm{mL}, 75$ th percentile $8.23 \mu \mathrm{g} / \mathrm{mL})$. Eighteen cases were catatonic, 43 cases were restrained (19 for 1-6 days, 24 for more than 7 days), and 12 cases had lower-extremity restraint (4 for 1-6 days, 8 for more than 7 days). Fiftyfive cases received VTE prevention treatment (47 for GCS without IPC, 8 for IPC), and no patients received heparin prophylaxis for VTE prevention. Six cases had a history of VTE. The ICD-10 diagnoses were as follows: 31 were F2, 43 were F3, and 27 were in other categories. Fifty-two cases were administered antipsychotics (CPZE 0-300 mg/day for 26, CPZE 300-600 mg/day for 12, CPZE $600 \mathrm{mg} /$ day for 14). A total of 35 cases were administered antidepressants (IMP 0-150 mg/day for 23 and IMP $150 \mathrm{mg}$ /day for 12). Five cases had malignancy, 4 cases had surgery within 4 weeks, 6 cases had trauma within 4 weeks, and 15 cases had diabetes. No patients were pregnant. Neither oral contraceptives nor hormone drugs were used.

The clinical characteristics of VTE-positive and VTEnegative patients are shown in Table 2. Compared to the VTE-negative group, the VTE-positive group was significantly more likely to be catatonic $(P=0.031)$. There was no significant difference between the 2 groups for age, sex, BMI, duration of restraint, duration of restraint of the lower extremities, prevention of VTE, history of VTE, diagnosis of psychiatric disorder, dosage of antipsychotics, dosage of antidepressants, malignancy, surgery within 4 weeks, trauma within 4 weeks, or diabetes. D-dimer value in the VTE-positive group was $7.570 \mu \mathrm{g} / \mathrm{mL}$ (25th percentile $5.265 \mu \mathrm{g} / \mathrm{mL}$, 75th percentile $13.945 \mu \mathrm{g} / \mathrm{mL}$ ) and $4.780 \mu \mathrm{g} / \mathrm{mL}$ (25th percentile $2.837 \mu \mathrm{g} / \mathrm{mL}$, 75th percentile $6.725 \mu \mathrm{g} / \mathrm{mL}$ ) in the VTE-negative group. Compared to D-dimer values in the VTE-negative group, D-dimer values in the VTE-positive group were significantly higher $(P<0.001)$. The results of the multiple logistic regression analysis are shown in Table 3. It demonstrated that catatonia was significantly associated with an increased incidence of VTE after adjusting for age, sex, catatonia, and duration of restraint (odds ratio $3.01 ; 95 \%$ CI 1.01-8.92, $P=0.047$ ). 
Table 2 Clinical and demographic characteristics of VTE-positive and VTE-negative patients

\begin{tabular}{|c|c|c|c|}
\hline $\begin{array}{l}\text { Patients underwent contrast- } \\
\text { enhanced } C T\end{array}$ & $\begin{array}{l}\text { VTE-positive, } \\
\mathbf{N}=39\end{array}$ & $\begin{array}{l}\text { VTE-negative, } \\
\mathrm{N}=62\end{array}$ & $P$-value \\
\hline Age (years) ${ }^{\mathrm{a}}$ & & & 0.50 \\
\hline$<65$ & 20 & 36 & \\
\hline$\geq 65$ & 19 & 26 & \\
\hline Sex ${ }^{a}$ & & & 0.22 \\
\hline Male & 11 & 25 & \\
\hline Female & 28 & 37 & \\
\hline $\mathrm{BMI}^{\mathrm{a}}$ & & & 0.61 \\
\hline$<18.5$ & 8 & 15 & \\
\hline$\geq 18.5$ and $<25.0$ & 19 & 24 & \\
\hline$\geq 25.0$ & 12 & 23 & \\
\hline Catatonia $^{a}$ & & & $0.031^{*}$ \\
\hline Absent & 28 & 55 & \\
\hline Present & II & 7 & \\
\hline Days of restraint ${ }^{a}$ & & & 0.41 \\
\hline 0 & 24 & 34 & \\
\hline $1-6$ & 4 & 15 & \\
\hline$\geq 7$ & 11 & 13 & \\
\hline Days of restraint of lower extremities ${ }^{b}$ & & & 0.25 \\
\hline 0 & 35 & 54 & \\
\hline $1-6$ & 0 & 4 & \\
\hline$\geq 7$ & 4 & 4 & \\
\hline Prevention of VTE ${ }^{\mathrm{b}}$ & & & 0.39 \\
\hline None & 17 & 29 & \\
\hline GCS without IPC & 17 & 30 & \\
\hline IPC & 5 & 3 & \\
\hline History of VTE ${ }^{\mathrm{b}}$ & & & 0.67 \\
\hline Absent & 36 & 59 & \\
\hline Present & 3 & 3 & \\
\hline$I C D-10^{a}$ & & & 0.28 \\
\hline F2 & 13 & 18 & \\
\hline F3 & 19 & 24 & \\
\hline Others & 7 & 20 & \\
\hline Antipsychotics dosage, CPZE mg/day ${ }^{\mathrm{a}}$ & & & 0.42 \\
\hline 0 & 16 & 33 & \\
\hline$>0$ and $<300$ & 10 & 16 & \\
\hline$\geq 300$ and $<600$ & 5 & 7 & \\
\hline$\geq 600$ & 8 & 6 & \\
\hline Antidepressants dosage, IMPE mg/day & & & 0.53 \\
\hline 0 & 23 & 43 & \\
\hline$>0$ and $<150$ & 11 & 12 & \\
\hline$\geq 150$ & 5 & 7 & \\
\hline Malignancy ${ }^{b}$ & & & 1.00 \\
\hline Absent & 37 & 59 & \\
\hline Present & 2 & 3 & \\
\hline Surgery within 4 weeks ${ }^{\mathrm{b}}$ & & & 0.64 \\
\hline Absent & 37 & 60 & \\
\hline Present & 2 & 2 & \\
\hline Trauma within 4 weeks ${ }^{\mathrm{b}}$ & & & 0.40 \\
\hline Absent & 38 & 57 & \\
\hline Present & I & 5 & \\
\hline Diabetes $^{\mathrm{a}}$ & & & 0.65 \\
\hline Absent & 34 & 52 & \\
\hline Present & 5 & 10 & \\
\hline
\end{tabular}

Notes: Values are presented as number. P-values with significant results are labeled with an asterisk. ${ }^{\mathrm{a} C h i-s q u a r e d}$ test. ${ }^{\mathrm{b}} \mathrm{Cisher}$ 's exact test.

Abbreviations: BMI, body mass index; CPZE, chlorpromazine equivalents; GCS, graduated compression stockings; ICD-I0, International Classification of Diseases I0th Revision; IMPE, imipramine equivalent; IPC, intermittent pneumatic compression; VTE, venous thromboembolism. 
Table 3 Predictive factors for VTE

\begin{tabular}{|c|c|c|c|c|}
\hline Variables & VTE-positive & $\begin{array}{l}\text { Odds } \\
\text { ratio }\end{array}$ & $95 \% \mathrm{Cl}$ & $P$-value \\
\hline \multicolumn{5}{|l|}{ Age } \\
\hline$<65$ & & I & & \\
\hline$\geq 65$ & & 1.28 & $0.52-3.14$ & 0.48 \\
\hline \multicolumn{5}{|l|}{ Sex } \\
\hline Male & & I & & \\
\hline Female & & 1.56 & $0.62-3.89$ & 0.59 \\
\hline \multicolumn{5}{|l|}{ Catatonia } \\
\hline Absent & 28/83 (33.7\%) & I & & \\
\hline Present & II/I8 (6I.1\%) & 3.01 & $1.01-8.92$ & $0.047^{*}$ \\
\hline \multicolumn{5}{|c|}{ Days of restraint } \\
\hline 0 & & I & & \\
\hline $1-6$ & & 0.41 & $0.12-1.45$ & 0.17 \\
\hline$\geq 7$ & & 1.37 & $0.48-3.91$ & 0.56 \\
\hline
\end{tabular}

Notes: Values are presented as numbers. $P$-values with significant results $(<0.05)$ are labeled with an asterisk.

Abbreviation: VTE, venous thromboembolism.

\section{Discussion}

This is the first systematic study investigating VTE including PE in psychiatric inpatients. Throughout the study period, VTE was observed in $2.3 \%$ of hospitalized patients, in $61.1 \%$ of catatonic patients, in $4.1 \%$ of noncatatonic restrained patients, and in $1.2 \%$ of noncatatonic unrestrained patients. This study also indicates that catatonia was associated with an increased incidence of VTE. Previous reports have described a $25.3 \%$ incidence of DVT in catatonic cases. ${ }^{6}$ In comparison, our study showed a very high incidence of VTE (61.1\%) in catatonic cases. It is possible that this seemingly high VTE percentage may be related to the following factors specific to this study: the incidence of PE that could not be detected by lower limb ultrasonography was high $44.4 \%$ (8/18), contrastenhanced CT has a higher diagnostic rate of DVT compared to lower limb ultrasonography, ${ }^{10}$ and heparin prophylaxis for prevention of $\mathrm{VTE}^{11}$ was not performed in the current study. Previous studies have reported a DVT incidence of $11.6 \%$ in restrained patients, ${ }^{5}$ as compared to the low value of $4.1 \%$ observed for VTE in this study. The reason may be due to the following: D-dimer was measured in $60.7 \%$ (164/270) of cases of noncatatonic restrained patients and contrast-enhanced CT examination was performed in $31.3 \%$ (36/115) of cases of abnormal D-dimer values. For noncatatonic unrestrained inpatients, there have been no previous research reports. In the current study, VTE was observed in $1.2 \%$ of cases; however, VTE screening was performed at a rate of only $17.9 \%(249 / 1,393)$, and VTE incidence could actually have been higher. According to a report from the USA, the annual incidence of VTE was 117 per 100,000 persons, ${ }^{12}$ but the results of the current study indicate a higher incidence. Additionally, several reports have indicated that the incidence of VTE in Japanese people is comparatively lower than that in Americans, ${ }^{13-15}$ with an increased risk of VTE in Americans.

VTE was observed in $38.6 \%$ of patients for whom contrast-enhanced CT was performed, $61.1 \%$ of catatonic patients in whom contrast-enhanced $\mathrm{CT}$ had been performed, $30.6 \%$ of noncatatonic restrained patients in whom contrastenhanced CT had been performed, and $36.2 \%$ of noncatatonic unrestrained patients in whom contrast-enhanced CT had been performed. However, careful interpretation of these results is necessary. The possibility exists that patients subjected to contrast-enhanced CT were at high risk for VTE and the selection bias was doubled for the performance of D-dimer measurement and contrast-enhanced CT in noncatatonic restrained and noncatatonic unrestrained cases of VTE. Prospective studies of hospitalized psychiatric patients in whom contrast-enhanced CT is used for the diagnosis of VTE and the assessment of the incidence of VTE (PE, DVT each) are warranted.

Two features of VTE in this study are noteworthy. First, of the VTE patients in this study, PE was observed in $76.9 \%$ (30/39). It is also important that 48.7\% (19/39) of VTE patients had PE without DVT. As PE cannot be diagnosed from a lower limb venous ultrasound, it would be missed if contrast-enhanced CT is not performed. However, as almost all patients were asymptomatic for PE, it is still unclear which patients should be checked by contrast-enhanced CT if D-dimer levels are abnormal. The reasons are that there is only 1 study on lung cancer patients compared between intervention group and non-intervention group for the prognosis of asymptomatic PE, ${ }^{16}$ and side effects such as anaphylactic shock were observed with contrast media. ${ }^{17}$ Second, the incidence of proximal DVT in DVT-positive patients was $55.0 \%(11 / 20)$ in this study. The incidence of proximal DVT in the current study was much higher than other reports on the incidence of proximal DVT., 5 In previous studies, the incidence of proximal DVT was reported as $13.3 \%(2 / 15)$ of catatonic patients ${ }^{6}$ and $0 \%(0 / 21)$ of restrained patients. ${ }^{5}$ It is thought that the higher diagnostic rate of proximal $\mathrm{DVT}^{10}$ observed in the current study than in the previous studies may be due to the use of contrast-enhanced CT, which has a higher diagnostic rate of asymptomatic DVT compared to lower limb ultrasonography, ${ }^{10}$ and facilitates diagnosis of DVT within the pelvic region, which is inaccessible to lower limb venous ultrasonography. It is reported that the severity of PE is related to the size of the embolus, and the severe PE cases are more common in proximal DVT than distal DVT. ${ }^{18,19}$ 
However, as for PE, it is still unclear whether contrastenhanced CT should be performed in all cases where D-dimer levels are high. In the present circumstances, whether contrast $\mathrm{CT}$ is performed or not on a patient with abnormal D-dimer levels but no DVT lower limb ultrasonography is a decision for the clinician based on the risk of proximal DVT.

There are differences in the cutoff values of the various reagents based on VTE screening D-dimer used in previous studies. ${ }^{20,21}$ NANOPIA D-dimer was used to measure D-dimer in the current research. ${ }^{8}$ This reagent has a cutoff value of $1.0 \mu \mathrm{g} / \mathrm{mL}$; however, the cutoff value of VIDAS ${ }^{\circledR}$ D-dimer (BioMérieux, Durham, NC, USA), which is commonly used in Europe and the USA, is $0.5 \mu \mathrm{g} / \mathrm{mL}$, resulting in the need for careful analysis across studies.

The strength of this study was that all cases underwent contrast-enhanced CT for diagnosis of VTE, and thus PE and DVT within the pelvis were detected, which would not have been the case with lower limb venous ultrasound.

The limitations of this study include 1) the retrospective nature, 2) lack of clearly defined criteria for the measurement of D-dimer, 3) presence of cases in which contrast-enhanced CT was not performed even though D-dimer values were abnormal, 4) no systematic investigation of the Wells score (DVT version) ${ }^{22}$ or Wells Score (PE version), ${ }^{23}$ or of the indicators of the clinical severity of acute pulmonary thromboembolism, ${ }^{24}$ or 5) lack of other possible risk factors for VTE such as hypercholesterolemia or smoking. ${ }^{25}$ 6) As there is little research on the cutoff value for the NANOPIA D-dimer in VTE screening, the value used $(1 \mu \mathrm{g} / \mathrm{mL})$ may be disputed.

\section{Conclusion}

The results indicate that the risk of VTE is high in all psychiatric inpatients regardless of the presence or absence of catatonia or restraint. Moreover, PE was found in $76.9 \%$ of VTE cases, which demonstrates the need for additional attention to this issue.

\section{Acknowledgments}

We would like to thank Editage (www.editage.jp) for English language editing. Dr Takeshima and Ishikawa served as cofirst authors for this work.

\section{Author contributions}

All authors contributed toward data analysis and revision of the manuscript and agree to be accountable for all aspects of this study.

\section{Disclosure}

The authors report no conflicts of interest in this work.

\section{References}

1. Malý R, Masopust J, Hosák L, Konupčiková K. Assessment of risk of venous thromboembolism and its possible prevention in psychiatric patients. Psychiatry Clin Neurosci. 2008;62(1):3-8.

2. Manu P, Kane JM, Correll CU. Sudden deaths in psychiatric patients. J Clin Psychiatry. 2011;72(7):936-941.

3. Zornberg GL, Jick H. Antipsychotic drug use and risk of first-time idiopathic venous thromboembolism: a case-control study. Lancet. 2000; 356(9237):1219-1223.

4. Barbui C, Conti V, Cipriani A. Antipsychotic drug exposure and risk of venous thromboembolism: a systematic review and meta-analysis of observational studies. Drug Saf. 2014;37(2):79-90.

5. Ishida $\mathrm{T}$, Katagiri $\mathrm{T}$, Uchida $\mathrm{H}$, et al. Incidence of deep vein thrombosis in restrained psychiatric patients. Psychosomatics. 2014;55(1):69-75.

6. Ishida T, Sakurai H, Watanabe K, Iwashita S, Mimura M, Uchida H. Incidence of deep vein thrombosis in catatonic patients: a chart review. Psychiatry Res. 2016;241:61-65.

7. American Psychiatric Association. Diagnostic and Statistical Manual of Mental Disorders. 5th ed. Arlington, VA, USA: American Psychiatric Publishing; 2013.

8. Yamaki T, Nozaki M, Sakurai H, et al. Combined use of pretest clinical probability score and latex agglutination D-dimer testing for excluding acute deep vein thrombosis. J Vasc Surg. 2009;50(5):1099-1105.

9. Kanda Y. Investigation of the freely available easy-to-use software 'EZR' for medical statistics. Bone Marrow Transplant. 2013;48(3):452-458

10. Kearon C, Julian JA, Newman TE, Ginsberg JS. Noninvasive diagnosis of deep venous thrombosis. McMaster diagnostic imaging practice guidelines initiative. Ann Intern Med. 1998;128(8):663-677.

11. Al Yami MS, Silva MA, Donovan JL, Kanaan AO. Venous thromboembolism prophylaxis in medically ill patients: a mixed treatment comparison meta-analysis. J Thromb Thrombolysis. 2018;45(1):36-47.

12. Silverstein MD, Heit JA, Mohr DN, Petterson TM, O'Fallon WM, Melton LJ 3rd. Trends in the incidence of deep vein thrombosis and pulmonary embolism: a 25-year population-based study. Arch Intern Med. 1998;158(6):585-593.

13. Hirst AE, Gore I, Tanaka K, Samuel I, Krishtmukti I. Myocardial infarction and pulmonary embolism. Arch Pathol. 1965;80:365-370.

14. Kumasaka N, Sakuma M, Shirato K. Incidence of pulmonary thromboembolism in Japan. Jpn Circ J. 1999;63(6):439-441.

15. Kitamukai O, Sakuma M, Takahashi T, Kagaya Y, Watanabe J, Shirato K. Incidence and characteristics of pulmonary thromboembolism in Japan 2000. Intern Med. 2003;42(11):1090-1094.

16. Sun JM, Kim TS, Lee J, et al. Unsuspected pulmonary emboli in lung cancer patients: the impact on survival and the significance of anticoagulation therapy. Lung Cancer. 2010;69(3):330-336.

17. Vogl TJ, Honold E, Wolf M, Mohajeri H, Hammerstingl R. Safety of iobitridol in the general population and at-risk patients. Eur Radiol. 2006;16(6):1288-1297.

18. Ohgi S, Tachibana M, Ikebuchi M, Kanaoka Y, Maeda T, Mori T. Pulmonary embolism in patients with isolated soleal vein thrombosis. Angiology. 1998;49(9):759-764.

19. Ro A, Kageyama N, Tanifuji T, Fukunaga T. Pulmonary thromboembolism: overview and update from medicolegal aspects. Leg Med (Tokyo). 2008;10(2):57-71.

20. Nomura H, Wada H, Mizuno T, et al. Negative predictive value of D-dimer for diagnosis of venous thromboembolism. Int $J$ Hematol. 2008;87(3):250-255.

21. Wells PS, Anderson DR, Rodger M, et al. Evaluation of D-dimer in the diagnosis of suspected deep-vein thrombosis. N Engl J Med. 2003; 349(13): 1227-1235.

22. Wells PS, Hirsh J, Anderson DR, et al. Accuracy of clinical assessment of deep-vein thrombosis. Lancet. 1995;345(8961):1326-1330.

23. Wells PS, Anderson DR, Rodger M, et al. Derivation of a simple clinical model to categorize patients probability of pulmonary embolism: increasing the models utility with the SimpliRED D-dimer. Thromb Haemost. 2000;83(3):416-420. 
24. Torbicki A, Perrier A, Konstantinides S, et al; ESC Committee for Practice Guidelines (CPG). Guidelines on the diagnosis and management of acute pulmonary embolism: the task force for the diagnosis and management of acute pulmonary embolism of the European Society of Cardiology (ESC). Eur Heart J. 2008;29(18):2276-2315.
25. Konstantinides SV, Torbicki A, Agnelli G, et al; Task Force for the Diagnosis and Management of Acute Pulmonary Embolism of the European Society of Cardiology (ESC). 2014 ESC guidelines on the diagnosis and management of acute pulmonary embolism. Eur Heart J. 2014;35(43):3033-3069.

\section{Publish your work in this journal}

Neuropsychiatric Disease and Treatment is an international, peerreviewed journal of clinical therapeutics and pharmacology focusing on concise rapid reporting of clinical or pre-clinical studies on a range of neuropsychiatric and neurological disorders. This journal is indexed on PubMed Central, the 'PsycINFO' database and CAS, and is the official journal of The International Neuropsychiatric Association (INA). The manuscript management system is completely online and includes a very quick and fair peer-review system, which is all easy to use. Visit http://www.dovepress.com/testimonials.php to read real quotes from published authors.

Submit your manuscript here: http://www.dovepress.com/neuropsychiatric-disease-and-treatment-journal 